\title{
BMJ Open Classification of osteoarthritis phenotypes by metabolomics analysis
}

\author{
Weidong Zhang, ${ }^{1}$ Sergei Likhodii, ${ }^{2}$ Yuhua Zhang, ${ }^{1}$ Erfan Aref-Eshghi, ${ }^{1}$ \\ Patricia E Harper, ${ }^{1}$ Edward Randell, ${ }^{2}$ Roger Green, ${ }^{1}$ Glynn Martin, ${ }^{3}$ \\ Andrew Furey, ${ }^{3}$ Guang Sun, ${ }^{4}$ Proton Rahman, ${ }^{4}$ Guangju Zhai ${ }^{1,5}$
}

To cite: Zhang W, Likhodii S, Zhang $\mathrm{Y}$, et al. Classification of osteoarthritis phenotypes by metabolomics analysis. BMJ Open 2014;4:e006286. doi:10.1136/bmjopen-2014006286

- Prepublication history and additional material is available. To view please visit the journal (http://dx.doi.org/ 10.1136/bmjopen-2014006286).

Received 1 August 2014 Revised 1 October 2014 Accepted 3 October 2014

For numbered affiliations see end of article.

Correspondence to Dr Guangju Zhai; guangju.zhai@med.mun.ca

\section{ABSTRACT}

Objectives: To identify metabolic markers that can classify patients with osteoarthritis (OA) into subgroups.

Design: A case-only study design was utilised.

Participants: Patients were recruited from those who underwent total knee or hip replacement surgery due to primary OA between November 2011 and December 2013 in St. Clare's Mercy Hospital and Health Science Centre General Hospital in St. John's, capital of Newfoundland and Labrador (NL), Canada. 38 men and 42 women were included in the study. The mean age was $65.2 \pm 8.7$ years.

Outcome measures: Synovial fluid samples were collected at the time of their joint surgeries. Metabolic profiling was performed on the synovial fluid samples by the targeted metabolomics approach, and various analytic methods were utilised to identify metabolic markers for classifying subgroups of patients with $O A$. Potential confounders such as age, sex, body mass index (BMI) and comorbidities were considered in the analysis.

Results: Two distinct patient groups, $A$ and $B$, were clearly identified in the 80 patients with $O A$. Patients in group A had a significantly higher concentration on 37 of 39 acylcarnitines, but the free carnitine was significantly lower in their synovial fluids than in those of patients in group $B$. The latter group was further subdivided into two subgroups, that is, B1 and B2. The corresponding metabolites that contributed to the grouping were 86 metabolites including 75 glycerophospholipids (6 lysophosphatidylcholines, 69 phosphatidylcholines), 9 sphingolipids, 1 biogenic amine and 1 acylcarnitine. The grouping was not associated with any known confounders including age, sex, BMI and comorbidities. The possible biological processes involved in these clusters are carnitine, lipid and collagen metabolism, respectively.

Conclusions: The study demonstrated that OA consists of metabolically distinct subgroups. Identification of these distinct subgroups will help to unravel the pathogenesis and develop targeted therapies for $\mathrm{OA}$.

\section{INTRODUCTION}

Osteoarthritis (OA) is a heterogeneous disease with various pathogenic factors and consists of different phenotypes which continually evolve, eventually leading to

\section{Strengths and limitations of this study}

- The strength of the study is the use of synovial fluid samples rather than plasma or urine samples. The metabolic profiling in synovial fluid reflects directly what is happening in a joint and yields the most accurate, real-time and joint-specific metabolic profile that is relevant to osteoarthritis (OA).

- $\mathrm{OA}$ is a heterogeneous disease. This is the first study that demonstrated that $\mathrm{OA}$ consists of at least three metabolically distinct subgroups, which are likely due to the differences in carnitine, lipid and collagen metabolism.

- The study was limited to only patients with $O A$, and we did not have synovial fluid samples from healthy people. A study with control synovial fluid samples is needed to confirm the findings.

common clinical and radiographic manifestations. ${ }^{1}$ Various classifications have been proposed depending on the main underlying pathophysiological mechanisms, ${ }^{2}$ clinically relevant patient characteristics, ${ }^{3}$ stage of disease, ${ }^{4}$ involved joints ${ }^{5}{ }^{6}$ and degree of inflammation. ${ }^{7}$ However, most patients could not fit easily into these proposed OA subgroups $^{2}$ due to the complexity of the influencing factors.

Recent studies have implied that $\mathrm{OA}$ is a metabolic disease linked to several components of the metabolic syndrome, such as hypertension, type-2 diabetes and dyslipidemia. ${ }^{8-10}$ Metabolites represent intermediate and end products of various cellular processes, whose levels can be regarded as a consequence of biological systems response to genotypic and environmental influences. Synovial fluid (SF) is an ultrafiltrate of plasma that also contains locally synthesised factors. Altered composition or concentrations of SF components are directly linked to OA. ${ }^{11}$ Using a metabolomics approach, we identified branched-chain amino acid to histidine ratio as a novel metabolic biomarker 
for knee OA. ${ }^{12} 13$ Other than biomarker identification, classification for a heterogeneous condition such as OA will require a reliable analytical method with good sensitivity and accuracy because the variation of the metabolite concentrations between phenotypes for a heterogeneous disease may be much narrower than that between people with and without the disease.

The Biocrates AbsoluteIDQ p180 kit is a commercially available product for targeted metabolomics which can simultaneously identify and quantify 186 metabolites from 5 different compound classes. The assay is performed using a combined ultra performance liquid chromatography (UPLC) and mass spectrometry-based flow injection analysis (FIA) method which has proven to be in conformance with the Food and Drug Administration (FDA) Guideline "Guidance for Industry -Bioanalytical Method Validation". ${ }^{14}$ Compared with untargeted screening, the multiple reaction monitoring (MRM) mode was adopted in this method which can offer better sensitivity and quantitative accuracy for analysis. Our previous study ${ }^{12}$ has demonstrated it to be a reliable and sensitive method for metabolite detection. In the present study, we used the p180 kit to identify metabolic markers in SFs that can be used to classify patients with OA into distinct subgroups.

\section{PATIENTS AND METHODS}

\section{Patients}

The present study was part of the Newfoundland Osteoarthritis Study (NFOAS) that was initiated in 2011 and aimed at identifying novel genetic, epigenetic and biochemical markers for OA. ${ }^{15}$ Patients with OA were recruited from those who underwent total knee or hip replacement surgery due to primary OA between November 2011 and December 2013 in St. Clare's Mercy Hospital and Health Science Centre General Hospital in St. John's, the capital city of Newfoundland and Labrador (NL), Canada. The response rate was $90 \%$. OA diagnosis was performed based on the American Rheumatology College's criteria and the judgement of the attending orthopaedic surgeon.

\section{Demographic and comorbidities}

Demographic and medical information was collected by a self-administered general questionnaire with the assistance of research staff if necessary. Age was calculated at the time of surgery. Comorbidities including eight metabolic-related diseases (heart disease, hypertension, high blood pressure in pregnancy, high cholesterol, diabetes, stroke, gout and osteoporosis) were confirmed by reviewing the electronic hospital records, which contain relevant clinical, laboratory and radiographic information. Height and weight measurements were obtained from the patient's hospital medical records. BMI was calculated as weight in kilograms divided by the squared height in metres.

\section{Metabolic profiling}

SFs were collected during the joint surgeries. Prior to knee arthrotomy/hip capulotomy, a syringe was inserted into the suprapatellar pouch of the knee/hip along the femoral neck, and 2-4 mL of the SF samples was aspirated. The samples were then put in vials and stored in liquid nitrogen until analysis. Metabolic profiling was performed by using the Waters XEVO TQ MS system (Waters Limited, Mississauga, Ontario, Canada) coupled with the Biocrates AbsoluteIDQ p180 kit, which measures 186 metabolites including 90 glycerophospholipids, 40 acylcarnitines ( 1 free carnitine), 21 amino acids, 19 biogenic amines, 15 sphingolipids and 1 hexose $(>90 \%$ is glucose). The details of these 186 metabolites are listed in online supplementary table S1. The metabolic profiling method using this kit was described previously. $^{12}$

\section{Statistical methods}

Data analyses encompassed hundreds of variables that are highly correlated. Dimension reduction was performed by multivariate methods that not only sought to capture changes of single metabolites between different groups, but also to utilise the dependency structures between the individual molecules. Principal component analysis (PCA), cluster analysis and partial least squares (PLS) regression, which are the most prominent multivariate analysis techniques applied in the research of metabolomics, ${ }^{16}$ were used in the analysis.

PCA is a statistical procedure that uses an orthogonal transformation to convert a set of observations of possibly correlated variables into a set of principal components. The first principal component has the largest possible variance, and each succeeding component in turn has the highest variance possible. ${ }^{17}$ Hierarchical cluster analysis (HCA) is also an unsupervised multivariate technique and was used to ${ }^{18}$ provide a visual description of the evolution of the clusters. Identification of the characteristic metabolites with significance between clusters was performed using the PLS-Discriminant Analysis (PLS-DA) method implemented in SIMCA-P 11.5 (Umetrics AB, Umea, Sweden) software. In PLS-DA, the R2X, R2Y and Q2 (cum) parameters were used for the model evaluation. $\mathrm{R} 2 \mathrm{X}$ is the percentage of all response variables explained by the model. R2Y is the percentage of all observation or sample variables explained by the model. Q2 is the percentage of all observation or sample variables predicted by the model. The importance of each metabolite in the PLS-DA was evaluated by variable importance in the projection (VIP) score. The VIP score positively reflects the metabolite's influence on the classification, and metabolites with a score greater than 1 were considered important in this study. Additionally, the Kruskal-Wallis test was executed using Multi Experiment View (V.4.9) software to determine the significant metabolites. The significance level was defined as $\mathrm{p}<0.01$. A heatmap was made using Multi Experiment View (V.4.9) software to present a detailed 
Table 1 Descriptive statistics of the study population*

\begin{tabular}{llll}
\hline & \multicolumn{3}{c}{$\mathbf{B}(\mathbf{n = 6 9 )}$} \\
\cline { 3 - 4 } Clusters & $\begin{array}{l}\mathbf{B} 1 \\
\mathbf{( n = 1 1 )}\end{array}$ & $\begin{array}{l}\mathbf{B} \mathbf{( n = 4 8 )} \\
\mathbf{( n = 2 1 )}\end{array}$ \\
\hline Age (years) & $67.2 \pm 5.8$ & $64.8 \pm 7.8$ & $65.3 \pm 11.8$ \\
Sex ratio (female \%) & 55 & 52 & 52 \\
BMI (kg/m $\left.{ }^{2}\right)$ & $35.9 \pm 5.7$ & $34.2 \pm 7.3$ & $29.6 \pm 5.3$ \\
Disease & & & \\
$\quad$ Heart disease (\%) & 0 & 8.3 & 9.5 \\
Hypertension (\%) & 27.2 & 47.9 & 33.3 \\
$\quad$ High blood pressure in & 0 & 4.2 & 4.8 \\
pregnancy (\%) & & & \\
$\quad$ High cholesterol (\%) & 27.2 & 39.6 & 28.6 \\
$\quad$ Diabetes (\%) & 18.2 & 12.5 & 23.8 \\
$\quad$ Stroke (\%) & 9.1 & 4.2 & 0 \\
$\quad$ Gout (\%) & 0 & 10.4 & 9.5 \\
$\quad$ Osteoporosis (\%) & 9.1 & 12.5 & 0 \\
\hline
\end{tabular}

${ }^{*}$ Data are presented as mean \pm SD unless indicated. BMI, body mass index.

description for each group. Discriminatory metabolites with these parameters are identified.

The above analyses were performed on concentrations obtained from the Absolute IDQ kit. Before analysis, raw data were filtered by the presence of metabolites in at least $80 \%$ of patients and all data were mean-centred and standardised.

\section{RESULTS}

A total of 38 men and 42 women were included in the study. The mean age was $65.2 \pm 8.7$ years, and the mean BMI was $33.3 \pm 6.9 \mathrm{~kg} / \mathrm{m}^{2}$. We had data on eight metabolic-related diseases including hypertension, dyslipidemia and diabetes that were previously reported to be associated with OA. ${ }^{10}$ The detailed descriptive statistics are presented in table 1.

Over $90 \%$ of the potential metabolites (168/186) were successfully determined in each sample. These included 40 acylcarnitines ( 1 free carnitine), 20 amino acids,
9 biogenic amines, 87 glycerophospholipids, 11 sphingolipids and 1 hexose ( $>90 \%$ is glucose). Since there were vast differences in the absolute concentrations among different metabolites, we standardised the concentration by using the Z-score for comparability between different metabolites for their biological relevance and used them in subsequent analyses.

Figure 1 presents the PCA results. Eighty patients with OA were clearly clustered into two distinct groups, that is, cluster A and cluster B (including several subassembling groups). Cluster A including 11 patients mainly assembled in the first quadrant, while cluster B consists of 69 patients scattered along the $\mathrm{X}$-axis. From the loading values, PC ae C40:1, PG ae C40:5, PG ae C36:1, PC ae C40:4 and PC ae C40:3 were the major contributors for component 1, whereas C12, C6:1, C3-OH, C3-DC (C4-OH), C3:1, C14:1 and C14 were the main contributors for component 2.

Using the HCA method, the patients of cluster B can be further classified into two subgroups, B1 and B2. It also appeared that group B1 could be divided into B1-1, B1-2-1 and B1-2-2 groups, and B2 could be subdivided into B2-1 and B2-2 groups, respectively (figure 2).

Identification of the metabolites with the most significantly different concentrations between groups was performed using the PLS-DA method. Data obtained from cluster A and cluster B were first used to construct a PLS-DA model, which showed the performance statistics of $\mathrm{R} 2 \mathrm{X}=0.603$, $\mathrm{R} 2 \mathrm{Y}=0.983$ and with a high prediction parameter Q2 (cum) of 0.98. The discriminant analysis showed that the concentrations of 37 acylcarnitines in cluster A were significantly higher than that in cluster B $(13.5 \pm 14.0$ fold for 34 of $37,309 \pm 261$ fold for 3 of 37 , all $\mathrm{p}<0.01$ ), while C0 (free carnitine, L-3-hydroxy-4aminobutyrobetaine) and C2 (acetylcarnitine) were significantly lower in Cluster A than that in cluster B (2.7 fold for $\mathrm{C} 2$, 3.1 fold for $\mathrm{C} 0$, all $\mathrm{p}<0.01$; figure 3 ). When the ratios of acylcarnitines to $\mathrm{C} 0$ was calculated, cluster A had far greater ratios than that of cluster B $(p<0.01)$. However, the total carnitine (acyl+free) level was not
Figure 1 The result of the principal component analysis.

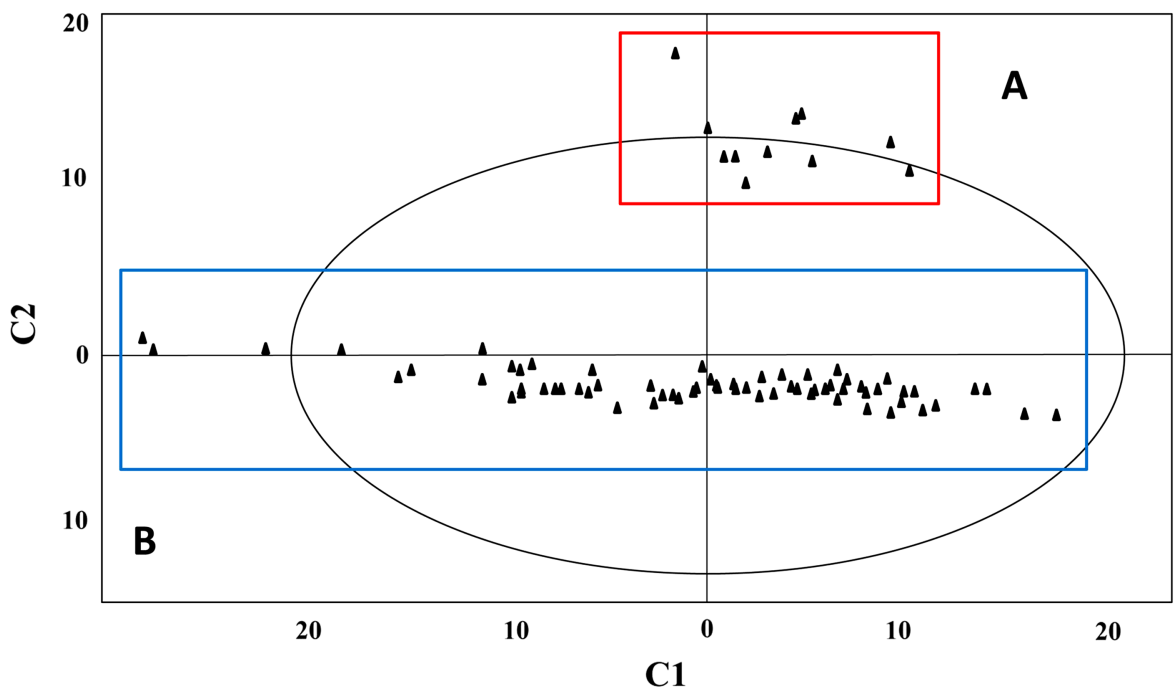




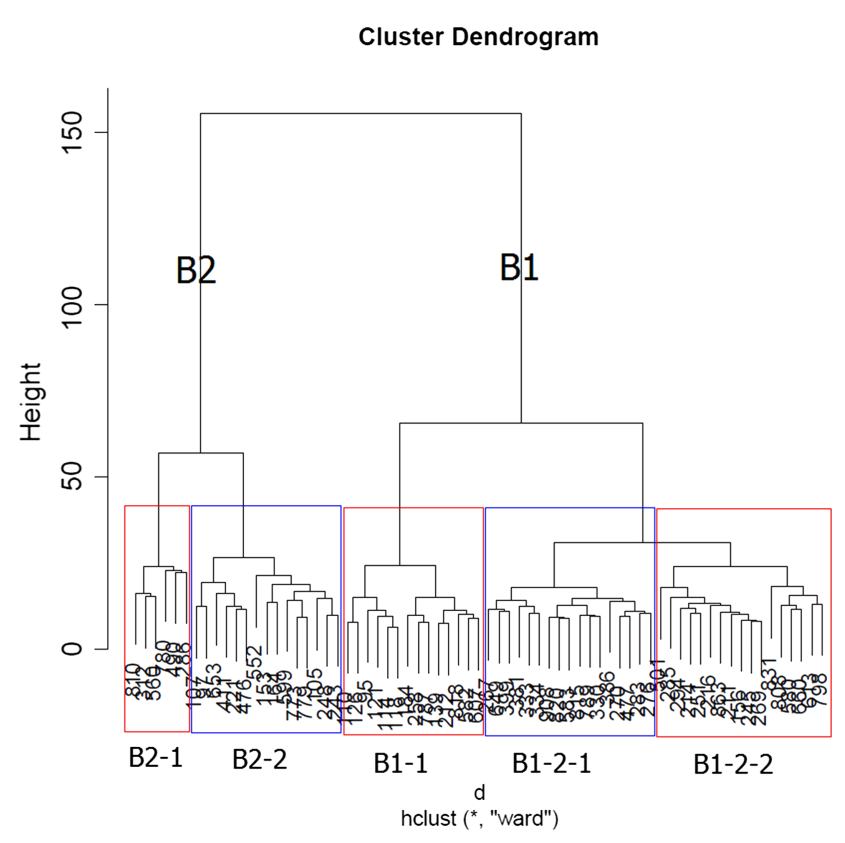

Figure 2 Hierarchical clustering analysis for group B (69 patients).

statistically significant between group A and group B $(\mathrm{p}=0.75)$.

Data were obtained from clusters B1 and B2 to construct a PLS-DA model with parameters: $\mathrm{R} 2 \mathrm{X}=0.546$, $\mathrm{R} 2 \mathrm{Y}=0.854$ and Q2 (cum) of 0.98. The discriminant analysis between clusters B1 and B2 was executed. A total of 86 metabolites including 75 glycerophospholipids (6 lysophosphatidylcholines, 69 phosphatidylcholines), 9 sphingolipids, 1 biogenic amine and 1 acylcarnitine were found to be significantly higher in cluster B2 than in cluster B1 by 1.58 fold, 1.83 fold, 1.72 fold, 1.19 fold and 1.82 fold, respectively (all $\mathrm{p}<0.01$ ). However, there are two acylcarnitines, C3 and C5, with a slightly lower concentration in B2 than in B1.

Specific metabolites that were distinct between B1-1, B1-2-1, B1-2-2, B2-1 and B2-2 were obtained by the
PLS-DA method with VIP $>1$ and $\mathrm{p}<0.01$, and the results are shown in figure 4 and online supplementary table S2. The significant metabolites include 14 amino acids, 24 glycerophospholipids, 12 acylcarnitines and 1 sphingolipid, in which proline had the biggest VIP value. Group B1-2-2 samples have a higher concentration in 12 amino acids and 1 biogenic amine than in the other four groups. Group B2-1 samples have the highest level of two amino acids (glutamate and ornithine) and all glycerophospholipids except two lyso-PC types (lyso-PC 16:0 and 16:1). On the whole, group B1-2-1 and group B2-2 have a compromised concentration in all significant metabolites and cluster B1-1 has the lowest concentration in all metabolites.

We explored whether the groupings were associated with any of the potential covariates. For groups A and B, the mean ages were $67.2 \pm 5.8$ years and $64.9 \pm 9$ years, respectively, but the difference was not statistically significant $(\mathrm{p}=0.42)$. Women made up $55 \%$ of group A and $52 \%$ of group B. The average BMI in group A was $35.9 \pm 5.7 \mathrm{~kg} /$ $\mathrm{m}^{2}$, which was higher than that in group B $(32.8 \pm 7.0 \mathrm{~kg} /$ $\mathrm{m}^{2}$ ), but the difference was not statistically significant $(p=0.17)$. We had data on eight metabolic-related diseases (heart disease, hypertension, high blood pressure in pregnancy, high cholesterol, diabetes, stroke, gout and osteoporosis) on all the study participants. The more prevalent diseases in these patients with OA were hypertension $(41.3 \%)$, high cholesterol (35\%) and diabetes $(16.3 \%)$. Although group A had a tendency of low prevalence of these metabolic-related diseases than group B, the differences were not statistically significant (all $\mathrm{p}>0.05$ ).

For the groups B1 and B2, there was no significant difference in age and sex between groups B1 and B2, but BMI in group B1 was higher than that in group B2 (34.2 \pm 7.2 vs $29.6 \pm 5.3,0.01<\mathrm{p}<0.05)$.

\section{DISCUSSION}

To the best of our knowledge, this is the first study that used the metabolomics approach to classify patients with
Figure 3 Heatmaps of significant metabolites for the separation of groups $A$ and $B$.

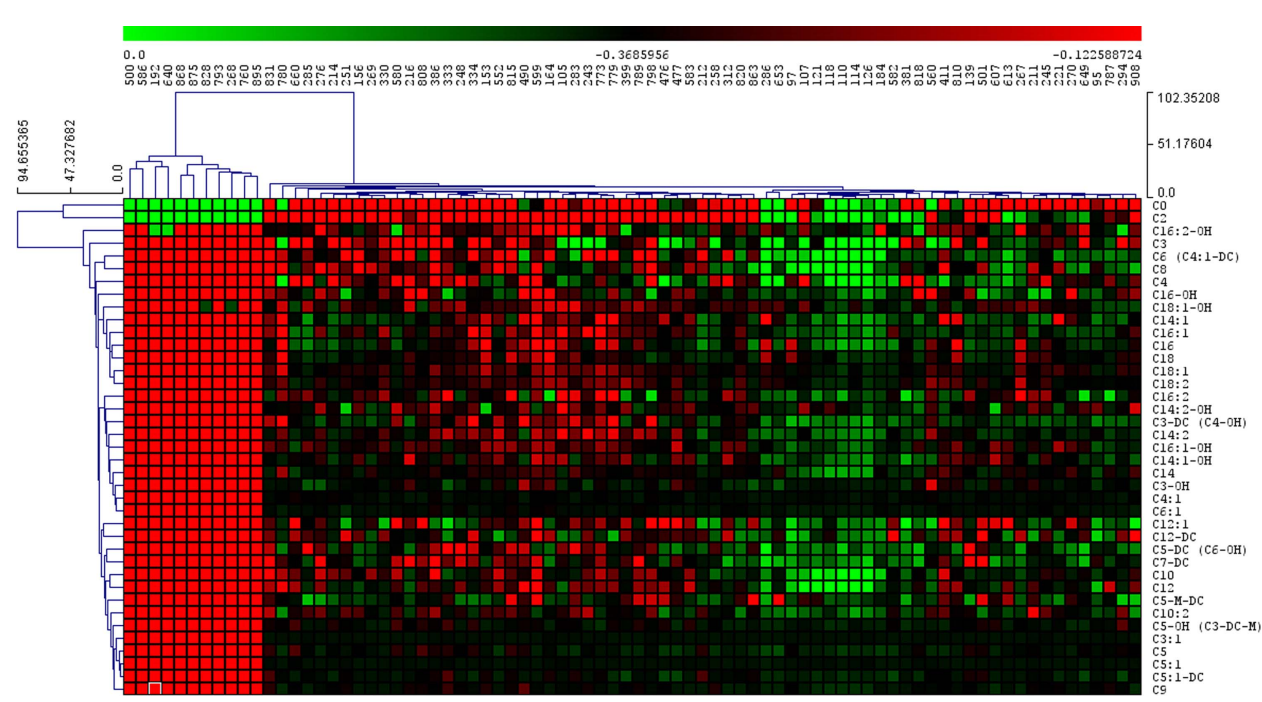


Figure 4 Heatmaps of significant metabolites for the separation of cluster $\mathrm{B} 1-1$. $\mathrm{B} 1-2-1, \mathrm{~B} 1-2-2, \mathrm{~B} 2-1$ and B2-2, $\mathrm{p}<0.01$.

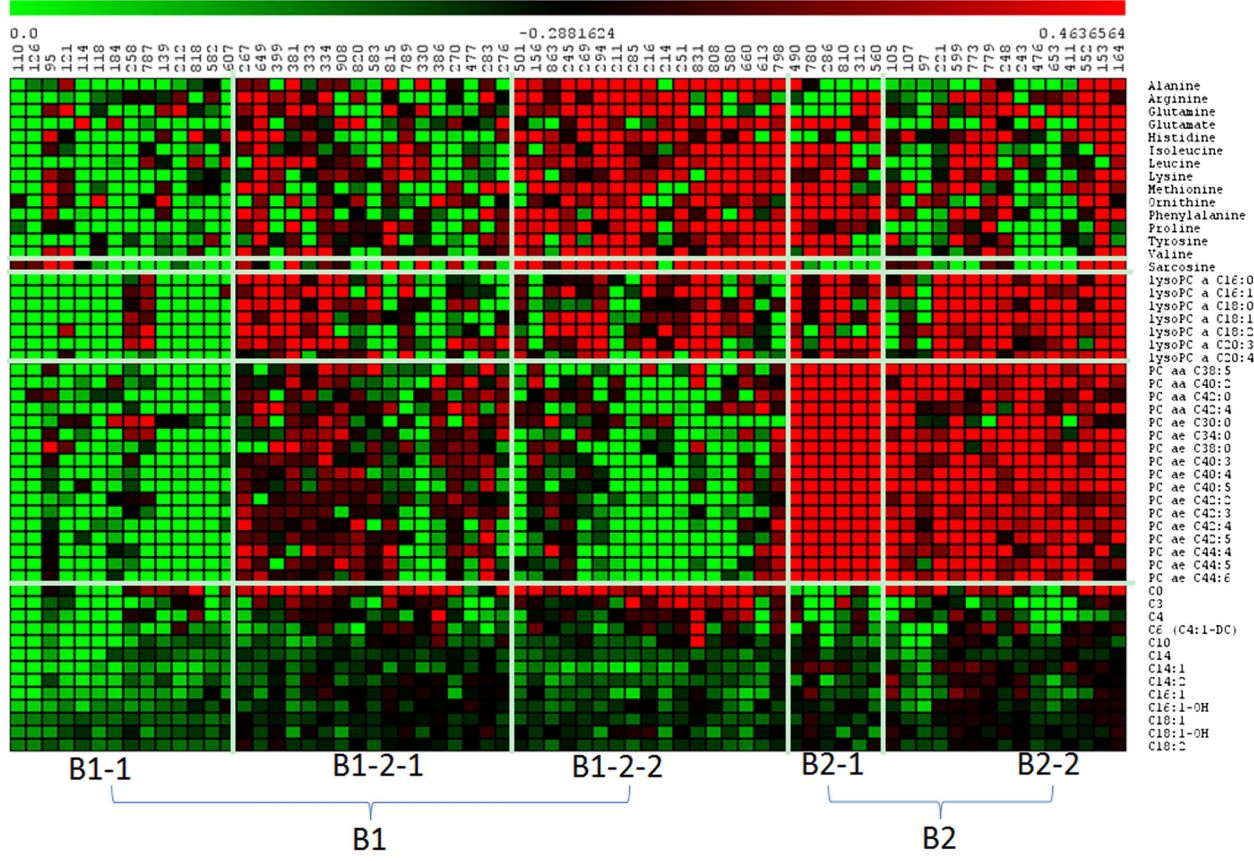

B1
OA into different distinct subgroups. The strength of the study is the use of SF samples rather than plasma or urine samples. Our unpublished data suggested that the metabolite correlation between plasma and SF was only modest (an average correlation coefficient of 0.22 ). Metabolic profiling in SF reflects directly what is happening in a joint and yields the most accurate, real-time and joint-specific metabolic profile that is relevant to OA. ${ }^{19}$

On the basis of metabolic profiling of the SFs, we classified 80 patients with OA into two large groups, that is, group A and group B. This is largely due to the difference in concentrations of acylcarnitines. Fasting might be a factor explaining the difference; however, all patients fasted on their surgery dates. When using the ratio of the concentrations of acylcarnitines to free carnitine $(\mathrm{C} 0)$, group A exclusively had greater ratios than did group B, indicating that two distinguished OA subphenotypes may be related to the carnitine metabolism pathway.

In humans, the major sources of camitine (C0) are de novo synthesis and the diet. In the process of synthesis of carnitine, glycine can be released as a by-product; ${ }^{20}$ however, there were no significant differences between both groups in the concentration of this metabolite. Carnitine acyltransferases are responsible for the production of acylcarnitines and the value of the acylcarnitines/carnitine ratio can reflect its activity. ${ }^{20} 21$ In the present study, the ratio of acylcarnitine to carnitine in group A is significantly higher than that of cluster B, which may indicate that the activity of acyltransferases is higher in patients of group A than group B. Carnitine and its acyl esters acylcarnitines are essential compounds for the metabolism of fatty acids. Carnitine can assist in the transport and metabolism of fatty acyl-CoA from the cytosol to the mitochondrial matrix, where the enzymes of $\beta$-oxidation are located and fatty acids are oxidised as a major source of energy. Inside the mitochondria, carnitine and acyl-CoA are regenerated, and the latter is catabolised in two-carbons units by $\beta$-oxidation, with production of acetyl-CoA in normal circumstances. Then the acetyl groups are converted to acetylcarnitine via the action of carnitine acetyltransferase for transport out of the mitochondria. In the patients of group A, both carnitine (C0) and acetylcarnitine (C2) are significantly lower than in patients in group B; however, the concentrations of other acylcarnitines are all significantly higher in group A than that in group B. This suggests that the activity of carnitine acetyltransferase is significantly lower in group B and there are differences in fatty acid metabolism between these two groups. Elevated acylcarnitine levels have been detected in obesity, ${ }^{22}$ type-2 diabetes, ${ }^{23}$ cardiovascular disease ${ }^{24}$ and encephalopathy. $^{25}$

Group B can be classified into group B1 and B2 based on the HCA. The significant metabolites that contribute to these subgroups are mainly glycerophospholipids, sphingolipids and amino acids

The physiological significance regarding OA for these kinds of metabolites has been previously studied. Glycerophospholipids form the essential lipid bilayer of all biological membranes and are intimately involved in signal transduction, regulation of membrane trafficking and many other membrane-related phenomena. ${ }^{26}{ }^{27}$ Studies by Hills ${ }^{28}$ indicated that alterations in phospholipid composition and concentrations are associated with the development of OA. Kosinska $e t a l^{29}$ also found that in comparison with control SF, the levels of glycerophospholipids (Five phosphatidylglycerol and two lysophosphatidylglycerol species) were all elevated in late OA by 3.6-fold. Our results are consistent with their findings. 
The concentrations of 24 glycerophospholipids in patients of group B2 were all significantly higher than those in patients of group B1, especially for the PC types.

Sphingolipids are a class of lipids that include ceramide species, sphingomyelins (SMs) and more complex glycosphingolipids, which are an important part of SF. Sphingolipids are structural components of plasma membranes and bioactive molecules that have significant functions in proliferation and growth as well as differentiation, cellular signal transduction and apoptosis in many mammalian cells, for instance, fibroblast-like synoviocytes and neural cells. ${ }^{30-33}$ Studies by Marta et $a l^{29}$ suggest that SM species had risen approximately twofold in SF from early OA to late OA. In our study, the concentration of nine significant sphingolipids (6 SM, $3 \mathrm{SM}$ $(\mathrm{OH}))$ in group B2 was significantly higher than that in group B1 by about 1.7 fold.

In metabolic disorders, the knowledge of the concentration of one amino acid or related group of amino acids is essential for correct diagnosis. For example, the cellular energy metabolism accessed by amino acids profiling can be used for in-depth analysis of chronic fatigue syndrome. ${ }^{34}$ The branched-chain amino acids (BCAA), valine, isoleucine and leucine, are essential amino acids, accounting for $35 \%$ of the essential amino acids in muscle proteins. On the basis of our previous studies, we believe it could be possible that an increased concentration of the BCAA leads to an increased production of cytokines, which then leads to an increased rate of joint collagen degradation. An increased level of cytokines has been associated with OA. ${ }^{12}{ }^{35}$ In the current study, some amino acids, including these three BCAA amino acids, were elevated in group B1-2-2, which could indicate that the patients with $\mathrm{OA}$ in this group were more likely concerned with collagen degradation. The proline, hydroxyproline and hydroxylysine levels are important indicators of connective tissue status. ${ }^{36}$ There were reports that proline incorporation into osteoarthritic cartilage was increased 4 -fold as compared to normal cartilage. ${ }^{37}$

Age, sex, BMI and comorbidities could be potential confounders. However, we did not find the grouping based on metabolic profiling in the present study to be associated with any of these potential confounders. Nevertheless, patients in group B tend to have a higher prevalence of hypertension than patients in group A. Liu et $a l^{38}$ using metabolomics analysis, showed significantly increased serum fatty acid levels in hypertension patients, which is consisted with our results.

There are some caveats. First, this is a case-only study and we do not have SF samples from healthy people. Although the metabolic map has presented a clear diversity character for patients with OA, metabolite concentrations in healthy people would provide a normal range so that we could distinguish which group has normal concentrations. Second, we do not have dietary and drug used information on the study participants, which might have an influence on metabolite concentrations, but the Newfoundland population is an isolated population characterised by relative environmental homogeneity and metabolite concentrations in SFs is less influenced by dietary intake. Third, we used a targeted metabolomics approach; thus, we might have missed important OA-associated metabolites which we were unable to measure. Lastly, our sample size was modest and a follow-up study with a large sample size is required to verify the findings.

\section{CONCLUSION}

This is the first study using a metabolomics approach to classify patients with OA and demonstrated that OA consists of metabolically distinct subgroups. While the findings need to be confirmed, the identification of these distinct subgroups will help to unravel the pathogenesis and develop targeted therapies for OA.

\section{Author affiliations}

${ }^{1}$ Discipline of Genetics, Faculty of Medicine, Memorial University of Newfoundland, St John's, Newfoundland, Canada

${ }^{2}$ Department of Laboratory Medicine, Faculty of Medicine, Memorial University of Newfoundland, St John's, Newfoundland, Canada

${ }^{3}$ Department of Surgery, Faculty of Medicine, Memorial University of Newfoundland, St John's, Newfoundland, Canada

${ }^{4}$ Discipline of Medicine, Faculty of Medicine, Memorial University of Newfoundland, St John's, Newfoundland, Canada

${ }^{5}$ Department of Twin Research \& Genetic Epidemiology, King's College London, London, UK

Acknowledgements The authors thank all the study participants who made this study possible, and all the staff in the hospital operation theatres who helped us in the collection of samples.

Contributors GZ conceptualised and designed the study, oversaw the sample collection, data analyses, and contributed to the drafting of the final manuscript; WZ contributed to metabolic profiling, data analyses and manuscript drafting; $Y Z$ contributed to the sample collection and data analyses; PEH, EA-E, GM and AF contributed to the sample collection and critical comments on the final manuscript; SL and ER contributed to the metabolic profiling, data analyses and critical comments on the final manuscript; RG, PR and GS contributed to the data collection and critical comments on the final manuscript. All authors approved the final manuscript as submitted.

Funding Canadian Institutes of Health Research (CIHR) (grant number RNL-132178); Newfoundland and Labrador RDC (grant number

5404.1423.102); Memorial University of Newfoundland (MUN); EA-E is supported by the Dean's PhD fellowship, Faculty of Medicine, MUN; PEH is supported by the CIHR CGS-Master's award.

\section{Competing interests None.}

\section{Patient consent Obtained.}

Ethics approval The study was approved by the Health Research Ethics Authority (HREA) of Newfoundland and Labrador and written consent was obtained from all the participants.

Provenance and peer review Not commissioned; externally peer reviewed.

Data sharing statement No additional data are available.

Open Access This is an Open Access article distributed in accordance with the Creative Commons Attribution Non Commercial (CC BY-NC 4.0) license, which permits others to distribute, remix, adapt, build upon this work noncommercially, and license their derivative works on different terms, provided the original work is properly cited and the use is non-commercial. See: http:// creativecommons.org/licenses/by-nc/4.0/ 


\section{REFERENCES}

1. Castañeda S, Roman-Blas JA, Largo R, et al. Osteoarthritis: a progressive disease with changing phenotypes. Rheumatology (Oxford) 2014;53:1-3.

2. Herrero-Beaumont G, Roman-Blas JA, Castañeda S, et al. Primary osteoarthritis no longer primary: three subsets with distinct etiological, clinical, and therapeutic characteristics. Semin Arthritis Rheum 2009;39:71-80.

3. Knoop J, van der Leeden M, Thorstensson CA, et al. Identification of phenotypes with different clinical outcomes in knee osteoarthritis: data from the Osteoarthritis Initiative. Arthritis Care Res (Hoboken) 2011;63:1535-42.

4. Scanzello CR, Umoh E, Pessler F, et al. Local cytokine profiles in knee osteoarthritis: elevated synovial fluid interleukin-15 differentiates early from end-stage disease. Osteoarthritis Cartilage 2009;17:1040-8.

5. Solovieva S, Kamarainen OP, Hirvonen A, et al. Association between interleukin 1 gene cluster polymorphisms and bilateral distal interphalangeal osteoarthritis. J Rheumatol 2009;36:1977-86.

6. MacGregor AJ, Li Q, Spector TD, et al. The genetic influence on radiographic osteoarthritis is site specific at the hand, hip and knee. Rheumatology (Oxford) 2009;48:277-80.

7. Haynes MK, Hume EL, Smith JB. Phenotypic characterization of inflammatory cells from osteoarthritic synovium and synovial fluids. Clin Immunol 2002;105:315-25.

8. Katz JD, Agrawal S, Velasquez M. Getting to the heart of the matter: osteoarthritis takes its place as part of the metabolic syndrome. Curr Opin Rheumatol 2010;22:512-19.

9. Bijlsma JW, Berenbaum F, Lafeber FP. Osteoarthritis: an update with relevance for clinical practice. Lancet 2011;377:2115-26.

10. Zhuo Q, Yang W, Chen J, et al. Metabolic syndrome meets osteoarthritis. Nat Rev Rheumatol 2012;8:729-37.

11. Ludwig TE, McAllister JR, Lun V, et al. Diminished cartilage-lubricating ability of human osteoarthritic synovial fluid deficient in proteoglycan 4: Restoration through proteoglycan 4 supplementation. Arthritis Rheum 2012;64:3963-71.

12. Zhai G, Wang-Sattler R, Hart DJ, et al. Serum branched-chain amino acid to histidine ratio: a novel metabolomic biomarker of knee osteoarthritis. Ann Rheum Dis 2010;69:1227-31.

13. Warde N. Osteoarthritis: Identification of a metabolomic biomarker for knee OA. Nat Rev Rheumatol 2010;6:381

14. U.S. Department of Health and Human Services FaDA, Center for Drug Evaluation and Research (CDER), Center for Veterinary Medicine (CVM) (2001) Guidance for Industry. Bioanalytical Method Validation.

15. Aref-Eshghi E, Rahman $\mathrm{P}$, Zhang H, et al. Attempt to replicate the published osteoarthritis-associated genetic variants in the Newfoundland \& Labrador Population. J Orthop Rheumatol 2014;1:5-10.

16. Bartel J, Krumsiek J, Theis FJ. Statistical methods for the analysis of high-throughput metabolomics data. Comput Struct Biotechnol J 2013;4:e201301009.

17. Lindon JC, Holmes E, Nicholson JK. Metabonomics techniques and applications to pharmaceutical research \& development. Pharml Res 2006;23:1075-88.

18. Beckonert O, Bollard ME, Ebbles TMD, et al. NMR-based metabonomic toxicity classification: hierarchical cluster analysis and k-nearest-neighbour approaches. Anal Chim Acta 2003;490:3-15.
19. Adams SB Jr, Setton LA, Nettles DL. The Role of Metabolomics in Osteoarthritis Research. J Am Acad Orthop Surg 2013;21:63-4.

20. Vaz FM, Wanders RJ. Carnitine biosynthesis in mammals. Biochem J 2002;361:417-29.

21. Gieger C, Geistlinger L, Altmaier E, et al. Genetics meets metabolomics: a genome-wide association study of metabolite profiles in human serum. PLOS Genet 2008;4:e1000282.

22. Ramos-Roman MA, Sweetman L, Valdez MJ, et al. Postprandial changes in plasma acylcarnitine concentrations as markers of fatty acid flux in overweight and obesity. Metabolism 2012;61:202-12.

23. Zammit VA, Ramsay RR, Bonomini M, et al. Carnitine, mitochondrial function and therapy. Adv Drug Deliv Rev 2009;61:1353-62.

24. Kalim S, Clish CB, Wenger J, et al. A plasma long-chain acylcarnitine predicts cardiovascular mortality in incident dialysis patients. J Am Heart Assoc 2013;2:e000542.

25. Murphy MG, Crocker JF, O'Regan P, et al. Carnitine, acylcarnitine and amino acid profiles analyzed by tandem mass spectrometry in a surfactant/virus mouse model of acute hepatic encephalopathy. Chemosphere 2007;68:1692-8.

26. Berridge MJ, Irvine RF. Inositol phosphates and cell signalling. Nature 1989;341:197-205.

27. Farooqui AA, Horrocks LA, Farooqui T. Glycerophospholipids in brain: their metabolism, incorporation into membranes, functions, and involvement in neurological disorders. Chem Phys Lipids 2000;106:1-29.

28. Hills BA. Surface-active phospholipid: a Pandora's box of clinical applications. Part II. Barrier and lubricating properties. Intern Med J 2002;32:242-51.

29. Kosinska MK, Liebisch G, Lochnit G, et al. Sphingolipids in human synovial fluid-a lipidomic study. PLoS ONE 2014;9:e91769.

30. Gerritsen ME, Shen CP, Perry CA. Synovial fibroblasts and the sphingomyelinase pathway: sphingomyelin turnover and ceramide generation are not signaling mechanisms for the actions of tumor necrosis factor-alpha. Am J Pathol 1998;152:505-12.

31. Cutler RG, Mattson MP. Sphingomyelin and ceramide as regulators of development and lifespan. Mech Ageing Dev 2001;122:895-908.

32. Spiegel S, Milstien S. Sphingosine-1-phosphate: an enigmatic signalling lipid. Nat Rev Mol Cell Biol 2003;4:397-407.

33. Luberto C, Kraveka JM, Hannun YA. Ceramide regulation of apoptosis versus differentiation: a walk on a fine line. Lessons from neurobiology. Neurochem Res 2002;27:609-17.

34. McGregor NR, Dunstan $\mathrm{RH}$, Zerbes $\mathrm{M}$, et al. Preliminary determination of the association between symptom expression and urinary metabolites in subjects with chronic fatigue syndrome. Biochem Mol Med 1996;58:85-92.

35. Livshits G, Zhai G, Hart DJ, et al. Interleukin-6 is a significant predictor of radiographic knee osteoarthritis: the Chingford Study. Arthritis Rheum 2009;60:2037-45

36. Kaddam IM, Iqbal SJ, Holland S, et al. Comparison of serum osteocalcin with total and bone specific alkaline phosphatase and urinary hydroxyproline:creatinine ratio in patients with Paget's disease of bone. Ann Clin Biochem 1994;3:327-30.

37. Lippiello L, Hall D, Mankin HJ. Collagen synthesis in normal and osteoarthritic human cartilage. J Clin Invest 1977;59:593-600.

38. Liu Y, Chen T, Qiu Y, et al. An ultrasonication-assisted extraction and derivatization protocol for GC/TOFMS-based metabolite profiling. Anal Bioanal Chem 2011;400:1405-17. 